\title{
On-Line Routing and Wavelength Assignment for Dynamic Traffic in WDM Ring and Torus Networks
}

\author{
Poompat Saengudomlert, Eytan H. Modiano, and Robert G. Gallager \\ Laboratory for Information and Decision Systems \\ Massachusetts Institute of Technology
}

\begin{abstract}
We develop on-line routing and wavelength assignment (RWA) algorithms for WDM bidirectional ring and torus networks with $N$ nodes. The algorithms dynamically support all $\mathrm{k}$-allowable traffic matrices, where $\mathrm{k}$ denotes an arbitrary integer vector $\left[k_{1}, k_{2}, \ldots, k_{N}\right]$, and node $i, 1 \leq i \leq N$, can transmit at most $k_{i}$ wavelengths and receive at most $k_{i}$ wavelengths. Both algorithms support the changing traffic in a rearrangeably nonblocking fashion. Our first algorithm, for a bidirectional ring, uses $\left\lceil\left(\sum_{i=1}^{N} k_{i}\right) / 3\right\rceil$ wavelengths in each ring direction and requires at most three lightpath rearrangements per new session request regardless of the number of nodes $N$ and the amount of traffic $\mathbf{k}$. When all the $k_{i}$ 's are equal to $k$, the algorithm uses $\lceil k N / 3\rceil$ wavelengths, which is known to be the minimum for any off-line rearrangeably nonblocking algorithm. Our second algorithm, for a torus topology, is designed for the special case with all the $k_{i}$ 's equal to $k$. For a square torus network with $N$ nodes, the algorithm uses $\lceil k \sqrt{N} / 2\rceil$ wavelengths in each fiber, which is shown to be at most two times a lower bound obtained by assuming full wavelength conversion at all nodes. In addition, the algorithm requires at most $\sqrt{N}-1$ lightpath rearrangements per new session request regardless of the amount of traffic $k$.
\end{abstract}

Index Terms-WDM networks, routing and wavelength assignment, graph theory.

\section{INTRODUCTION}

In a wavelength division multiplexed (WDM) network, the fiber bandwidth is divided into multiple frequency bands often called wavelengths. Using reconfigurable optical switches at the network nodes, some wavelengths can be selected at each node for termination and electronic processing, and others selected for optical bypass. In an all-optical network architecture, each traffic session optically bypasses electronic processing at each node on its path other than the source node and the destination node. One important benefit of this architecture is the cost saving resulting from using fewer and/or smaller elctronic switches in the network. We consider all-optical networks in this paper.

Without optical wavelength conversion, routing of traffic sessions is subjected to the wavelength continuity constraint, which dictates that the lightpath corresponding to a given session must travel on the same wavelength on all links from the source node to the destination node. Using wavelength converters potentially allows the network to support a larger set of traffic. However, such converters are likely to be expensive. Hence, several researchers have focused on the problem of routing and wavelength assignment (RWA) assuming no wavelength conversion. We shall focus on this same problem.

This work was supported by the National Science Foundation (NSF) under Grant ANI-0073730.
A large body of literature investigates the RWA problem under the wavelength continuity constraint. We can categorize existing results into two groups based on whether static or dynamic provisioning of routes and wavelengths is performed. For static provisioning, the traffic to be supported is assumed known and fixed over time. The goal is often to minimize the number of wavelengths used in the network [1], [2], or to maximize the number of supported traffic sessions for a fixed number of wavelengths [3], [4], [5], [6]. These problems are known to be NP-complete [3]. Consequently, bounds on the optimal costs have been derived [4], [7], and several RWA heuristics have been developed [1], [4], [5], [6], [8], [9].

Dynamic provisioning of routes and wavelengths gives us flexibility in supporting traffic which may change over time through session arrivals and session departures. To model dynamic traffic, session arrivals can be assumed to form stochastic processes [10], [11]. In addition, session lifetimes are stochastic. The goal is usually to develop an on-line RWA algorithm which minimizes the average blocking probability for a new session request given a fixed number of wavelengths in the network. We refer to this type of problem formulation as the blocking formulation. Due to the complexity in computing blocking probabilities, some approximations are made to simplify the analysis. For example, session arrivals on different links are assumed to be independent [10], [12], or correlated among adjacent links in the same fashion throughout the network [11]. Based on such approximations, several dynamic RWA heuristics have been developed [13], [14].

Another type of problem formulation, referred to as the nonblocking formulation, assumes prior knowledge of the set of all the traffic matrices, or equivalently the traffic demands, to be supported [15], [16], [17], [18]. In [16], the set of traffic matrices is characterized by the maximum link load in the network. In [15], [17], [18], the set of traffic matrices is characterized by the numbers of tunable transmitters and tunable receivers at each end node, i.e. a node which sources and/or sinks traffic sessions. A new session is said to be allowable if its arrival results in a traffic matrix which is still in the set of supportable traffic. The goal is usually to develop an on-line RWA algorithm which does not block any allowable session and uses the minimum number of wavelengths.

If we allow some existing lightpaths to be rearranged in order to support a new session, the corresponding RWA algorithm is said to be rearrangeably nonblocking. If we allow no rearrangement of any existing lightpath in order to support a new session, the corresponding RWA algorithm is said to be wide-sense nonblocking. Note that if a RWA algorithm is widesense nonblocking, it is also rearrangeably nonblocking. Thus, 
for the same set of traffic matrices, the required number of wavelengths can be no smaller for a wide-sense nonblocking RWA algorithm than for a rearrangeably nonblocking RWA algorithm.

In this paper, we investigate the RWA problem for dynamic traffic. We shall adopt a nonblocking formulation of the RWA problem. As in [15], [17], [18], the supportable traffic set is defined by the number of tunable transmitters and tunable receivers at each end node. We model the traffic as a sessionby-session arrival and departure process in which sessions arrive and depart one at a time, and each session utilizes a full wavelength. Our goal is to design an on-line RWA algorithm which is rearrangeably nonblocking, uses the minimum number of wavelengths, and requires few rearrangements of existing lightpaths in order to support each new and allowable session. We shall focus on a bidirectional ring topology and a torus topology. Our on-line RWA algorithm for the bidirectional ring first appears in [18]. This paper provides a more complete treatment on the subject.

This paper is organized as follows. In section II, we define the set of $\mathbf{k}$-allowable traffic based on the number of tunable transmitters and tunable receivers at each end node, and formulate the RWA problem for $\mathrm{k}$-allowable traffic. In section III, we describe our on-line RWA algorithm for a bidirectional ring topology. Section IV contains our on-line RWA algorithm for a torus topology. Finally, we summarize the results and point out future research directions in section $\mathrm{V}$.

\section{Problem Formulation}

Consider an all-optical WDM network with no wavelength conversion. Adjacent nodes are connected by two fibers, one in each direction. In addition, all fibers contain the same number of wavelengths. Assume that each traffic session has a rate of one wavelength. At a given time, only one session can use a specific wavelength in a fiber, but multiple sessions can travel through the same node. Let $N$ be the number of end nodes in the network. Node $i, 1 \leq i \leq N$, is equipped with $k_{i}$ fully tunable transmitters and $k_{i}$ fully tunable receivers. Consequently, at any time, node $i$ can transmit at most $k_{i}$ wavelengths and receive at most $k_{i}$ wavelengths. Such a traffic matrix is said to belong to a set of $\mathbf{k}$-allowable traffic, where $\mathbf{k}=\left[k_{1}, k_{2}, \ldots, k_{N}\right]$.

We model dynamic traffic as a session-by-session arrival and departure process in which sessions arrive and depart one at a time. In other words, a transition from one traffic matrix to another is a result of either a single session arrival or a single session departure. A new session request is allowable if the resultant traffic matrix is still in the set of $\mathbf{k}$-allowable traffic. The definition implies that, for each new and allowable session request, there is a free transmitter at the source node and a free receiver at the destination node. For convenience, throughout the paper, a new session is assumed to be allowable unless it is explicitly stated otherwise.

We want to design an on-line RWA algorithm which supports $\mathbf{k}$-allowable traffic in a rearrangeably nonblocking fashion, uses the minimum number of wavelengths, and requires few rearrangements of existing lightpaths in order to support each new session request. Our algorithm will be centralized in nature. We assume that traffic does not change too frequently and the RWA algorithm always has correct knowledge of the current RWA in the network. In addition, we assume there is sufficient time for lightpath rearrangements between consecutive transitions of the traffic matrix.

We shall consider two regular topologies, a bidirectional ring topology and a torus topology. Figure 1 illustrates the two topologies. In either topology, each node is considered an end node, i.e. it sources and/or sinks traffic as well as passes intermediate traffic. Since a bidirectional ring topology is widely used, its investigation is an important practical problem. Although the torus topology may not be implemented in practice, its investigation should give us better understanding of the RWA problem for dynamic traffic in a more densely connected network in comparison to the sparsely connected bidirectional ring.

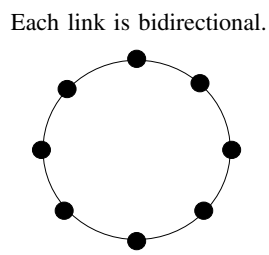

bidirectional ring topology

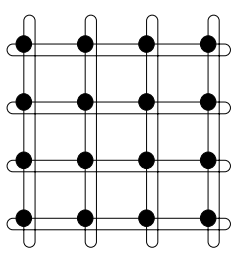

torus topology
Fig. 1. Bidirectional ring and torus topologies.

\section{BIDIRECTIONAL RING TOPOLOGY}

In this section, we study the RWA problem for $\mathrm{k}$-allowable traffic for an $N$-node bidirectional ring topology. Let $W_{\mathbf{k}}$ denote the minimum number of wavelengths which, if provided in each fiber, can support $\mathbf{k}$-allowable traffic with no wavelength conversion. Note that $W_{\mathbf{k}}$ is the number of wavelengths used to support any traffic matrix in the k-allowable set. Thus, for a specific traffic matrix, we may need fewer wavelengths than in the worst case. In [17], it was shown that, if all the $k_{i}$ 's are equal to $k$, then $W_{\mathbf{k}}=\lceil k N / 3\rceil$ for $N \geq 7 .^{1}$ In addition, an off-line RWA algorithm that uses at most $\lceil k N / 3\rceil$ wavelengths in each fiber, or equivalently in each ring direction, was developed.

We shall present an on-line RWA algorithm that uses $\left\lceil\left(\sum_{i=1}^{N} k_{i}\right) / 3\right\rceil$ wavelengths in each fiber to support $\mathbf{k}$ allowable traffic. Note that, for $N \geq 7$, when all the $k_{i}$ 's are equal to $k$, the algorithm uses the minimum number of wavelengths found in [17]. In all the other cases, the algorithm yields the upper bound $W_{\mathbf{k}} \leq\left\lceil\left(\sum_{i=1}^{N} k_{i}\right) / 3\right\rceil$.

Define a directed wavelength as a wavelength in either the clockwise or the counterclockwise ring direction. Given $w$ wavelengths in each fiber, there are $w$ directed wavelengths in the clockwise ring direction, and $w$ directed wavelengths in the counterclockwise ring direction. Note that any traffic session can be supported on a directed wavelength in either ring direction. Two sessions are said to be adjacent if the destination node of one session is the source node of the other session. The main idea behind our algorithm involves

\footnotetext{
${ }^{1}$ For $2 \leq N \leq 6$, the value of $W_{\mathbf{k}}$ can be found in [20].
} 
sharing a directed wavelength between two adjacent sessions, as suggested by the following known lemma in [17].

Lemma 1: In a bidirectional ring, lightpaths corresponding to any pair of adjacent sessions can either share a directed wavelength in the clockwise ring direction or the counterclockwise ring direction.

The proof of lemma 1 is immediate from figure 2, where if two lightpaths overlap in one direction, they do not overlap in the other direction. For example, lightpaths corresponding to a pair of adjacent sessions $(1,4)$ and $(4,2)$ collide in the clockwise ring direction, but do not collide in the counterclockwise ring direction. ${ }^{2}$ In what follows, when lightpaths associated with a pair of adjacent sessions share a directed wavelength, we simply say that the adjacent session pair share a directed wavelength.
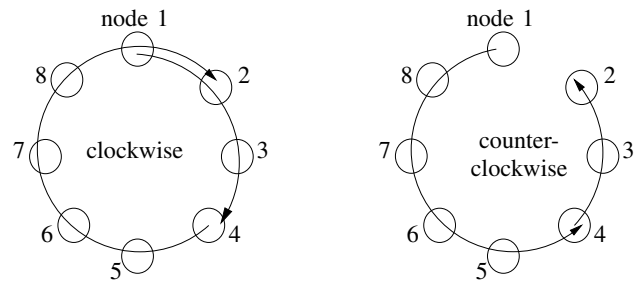

Fig. 2. Adjacent sessions share a directed wavelength.

The main idea of our algorithm is to maintain the following two RWA conditions at all times: $(i)$ only adjacent sessions share a directed wavelength, and $(i i)$ at most two adjacent sessions share a directed wavelength.

To give some intuition on the main idea of our algorithm, consider the special case with all the $k_{i}$ 's equal to 1 . In this case, our algorithm uses $\lceil N / 3\rceil$ wavelengths. We next describe informally how to use $\lceil N / 3\rceil$ wavelengths to support the traffic. We ignore integer rounding in the informal discussion below.

Given a traffic matrix, form as many adjacent session pairs as possible up to $N / 3$ pairs in a greedy fashion, i.e. it does not matter if we end up with less than the maximum possible number of pairs. Let $p$ denote the number of adjacent session pairs formed. Consider two cases.

Case 1: $p=N / 3$. In this case, we support $N / 3$ adjacent session pairs containing $2 N / 3$ sessions on $N / 3$ directed wavelengths in the required ring directions. This is always possible since there are $N / 3$ directed wavelengths available in each ring direction. Having done so, there are at most $N-2 N / 3=N / 3$ remaining sessions each of which we support on one directed wavelength in either ring direction. Thus, the total number of directed wavelengths required is at most $N / 3+N / 3=2 N / 3$. It follows that $N / 3$ wavelengths are sufficient.

Case 2: $p<N / 3$. In this case, we support $p$ adjacent session pairs containing $2 p$ sessions on $p$ directed wavelengths in the required ring directions. This is always possible since there are $N / 3$ directed wavelengths available in each ring direction. Note that we cannot form any more adjacent session pair in this case.

\footnotetext{
${ }^{2} \mathrm{~A}$ session from node $i$ to node $j$ is denoted by session $(i, j)$.
}

Consider only the sessions in the $p$ adjacent session pairs formed above. Define a common node to be a node which transmits a wavelength and receives a wavelength. Observe that each adjacent session pair has at least one common node. For example, figure 3 shows two $(p=2)$ adjacent session pairs $(7,4)$ and $(4,3)$ together with $(1,8)$ and $(8,7)$. The pair $(7,4)$ and $(4,3)$ has node 4 as a common node, while the pair $(1,8)$ and $(8,7)$ has node 8 as a common node. In addition, the sessions $(8,7)$ and $(7,4)$ make node 7 a common node. In general, given $p$ adjacent session pairs, there are at least $p$ common nodes.

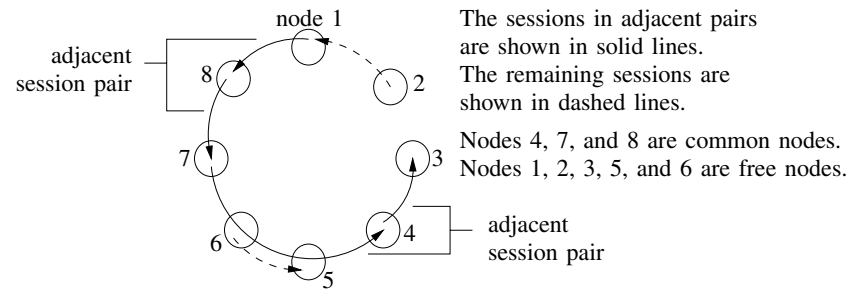

Fig. 3. Adjacent session pairs, common nodes, and free nodes.

Define a node which is not a common node as a free node. A free node still has a free transmitter and/or a free receiver. For example, in figure 3 , nodes $1,2,3,5$, and 6 are free nodes. Since there are at least $p$ common nodes, there are at most $N-p$ free nodes.

Consider the remaining sessions which are not in the $p$ adjacent session pairs formed above. Observe that each free node terminates, i.e. either transmits or receives, at most one remaining session. To see this, note that each free node cannot transmit more than one remaining session since it only has one transmitter. By the same argument, each free node cannot receive more than one remaining session. Moreover, each free node cannot transmit a remaining session and receive a remaining session simultaneously, or else we could form another new adjacent session pair, i.e. have more than $p$ pairs. Thus, each remaining session is terminated at two distinct free nodes. For example, in figure 3 , the remaining session $(2,1)$ is terminated at free nodes 1 and 2 . No other remaining session is terminated at either node 1 or node 2 . Since there are at most $N-p$ free nodes, there are at most $(N-p) / 2$ remaining sessions. We support each remaining session on one directed wavelength in either ring direction. Thus, the total number of directed wavelengths required is $p+(N-p) / 2=N / 2+p / 2<$ $N / 2+N / 6=2 N / 3$. It follows that $N / 3$ wavelengths are sufficient.

We shall later prove by similar arguments that $\left\lceil\left(\sum_{i=1}^{N} k_{i}\right) / 3\right\rceil$ wavelengths are sufficient to support $\mathbf{k}$ allowable traffic. We now describe our on-line RWA algorithm which is rearrangeably nonblocking, uses $\left\lceil\left(\sum_{i=1}^{N} k_{i}\right) / 3\right\rceil$ wavelengths in each fiber, and requires at most three lightpath rearrangements per new session request. We shall refer to this algorithm as the ring RWA algorithm.

Ring RWA Algorithm: (Use $\left\lceil\left(\sum_{i=1}^{N} k_{i}\right) / 3\right\rceil$ wavelengths.)

Session termination: When a session terminates, simply remove its associated lightpath from the ring without any 
further lightpath rearrangement.

Session arrival: When a session arrives and the resultant traffic matrix is still $\mathrm{k}$-allowable, proceed as follows.

Step 1: If there is a nonsharing session, i.e. a session which does not share its directed wavelength with any session, and it is adjacent to and can share its directed wavelength with the new session, assign the two sessions to share that directed wavelength. In this case, no lightpath rearrangement is required. Otherwise, proceed to step 2.

Step 2: If there is a free directed wavelength in either ring direction, assign a free directed wavelength to the new session. In this case, no lightpath rearrangement is required. Otherwise, proceed to step 3 .

Step 3: Among the nonsharing sessions and the new session, we claim and shall prove shortly that there must exist a pair of adjacent sessions. Form such an adjacent session pair by searching through all pairs of sessions in some order, e.g. from sessions terminating at node 1 to sessions terminating at node $N$. Once an adjacent session pair is found, there are two possibilities.

(3a) If the adjacent session pair can share the directed wavelength of one session in the pair, assign the adjacent session pair to share that directed wavelength. In this case, the adjacent session pair does not include the new session since step 1 would have otherwise applied. Therefore, one existing lightpath must be rearranged. Sharing of the directed wavelength by the adjacent session pair will free one directed wavelength on which the new session can be supported with only one lightpath rearrangement. Figure $4 \mathrm{a}$ illustrates this scenario. In particular, existing sessions $(1,5)$ and $(5,2)$ form an adjacent session pair which can be supported on the directed wavelength of session $(5,2)$. After the lightpath of session $(1,5)$ is rearranged, the new session $(1,4)$ is supported on the directed wavelength previously used by session $(1,5)$.

(3b) If the adjacent session pair cannot share the directed wavelength of either session in the pair, we claim and shall prove shortly that there must exist a directed wavelength with a nonsharing session in the opposite ring direction, i.e. the ring direction in which the adjacent session pair can share a directed wavelength. Remove the lightpath of that nonsharing session from its directed wavelength, and assign the adjacent session pair to share that directed wavelength. When the adjacent session pair includes the new session, the new session will now be supported, and sharing of the directed wavelength by the adjacent session pair will free one directed wavelength on which the removed nonsharing session can be supported. In this case, a total of two lightpath rearrangements are made. Figure $4 \mathrm{~b}$ illustrates this scenario. In particular, existing session $(1,5)$ and the new session $(5,2)$ form an adjacent session pair which can be supported on the directed wavelength of existing session $(3,8)$. After the lightpaths of sessions $(1,5)$ and $(3,8)$ are rearranged, the new session $(5,2)$ shares a directed wavelength with session $(1,5)$ on the directed wavelength previously used by session $(3,8)$, while session $(3,8)$ is supported on the directed wavelength previously used by session $(1,5)$.
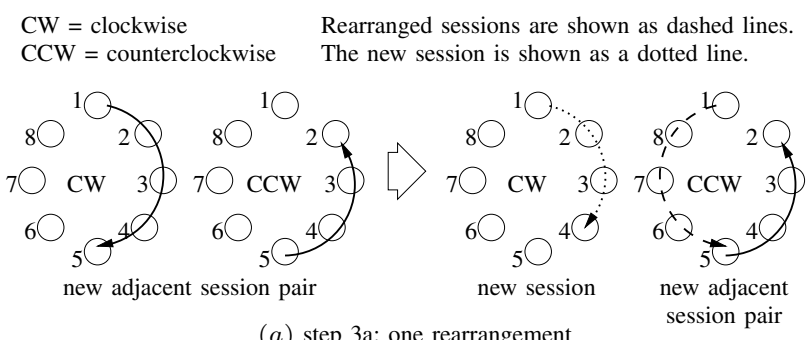

(a) step 3a: one rearrangement

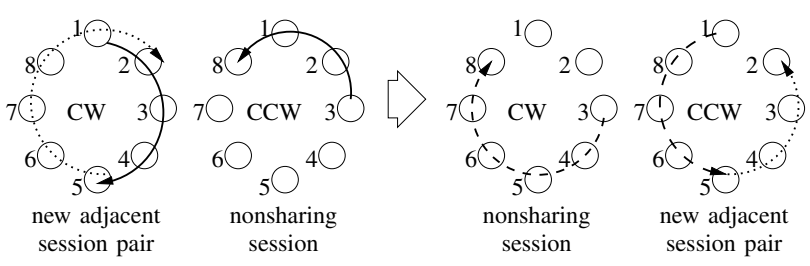

(b) step 3b case 1: two rearrangements

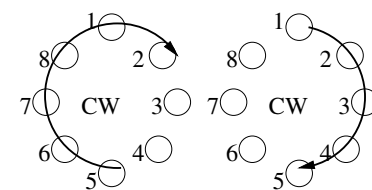

new adjacent session pair

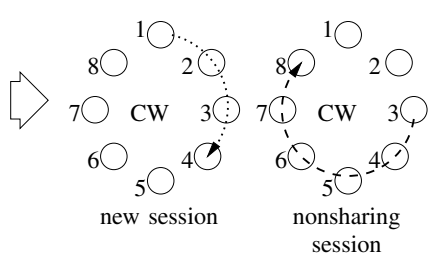

(c) step $3 \mathrm{~b}$ case 2: three rearrangement
When the adjacent session pair does not include the new session, sharing of the directed wavelength by the adjacent session pair will free two directed wavelengths on which the removed nonsharing session and the new session can be supported. In this case, a total of three lightpath rearrangements are made. Figure $4 \mathrm{c}$ illustrates this scenario. In particular, existing sessions $(1,5)$ and $(5,2)$ form an adjacent session pair which can be supported on the directed wavelength of existing session $(3,8)$. After the lightpaths of sessions $(1,5)$, $(5,2)$, and $(3,8)$ are rearranged, the adjacent session pair $(1,5)$ and $(5,2)$ are supported on the directed wavelength previously used by session $(3,8)$, session $(3,8)$ is supported on the directed wavelength previously used by session $(1,5)$, and the new session $(1,4)$ is supported on the directed wavelength previously used by session $(5,2)$.

Before proving the correctness of the ring RWA algorithm, we establish two useful lemmas related to step 3 of the algorithm. The first lemma gives an upper bound on the number of adjacent session pairs which share a directed wavelength in step 3 before the new session request. The second lemma gives an upper bound on the number of nonsharing sessions in step 3 before the new session request. In what follows, let $p$ be the number of adjacent session pairs which share a directed wavelength before the new session request. Let $q$ be the number of nonsharing sessions before the new session 
request. Let $w$ be the number of wavelengths in use before the new session request. Note that $w=p+q$. For convenience, define $K=\sum_{i=1}^{N} k_{i}$.

Lemma 2: In step 3 of the ring RWA algorithm, $p<$ $\lfloor K / 3\rfloor$.

Proof: Since the total number of sessions is at most $K$ in k-allowable traffic, it follows that $2 p+q<K$ before the new session request. Thus, $w$ is bounded by

$$
w=p+q<p+(K-2 p)=K-p .
$$

In step 3, since there is no free directed wavelength for the new session, it follows that the number of wavelengths in use $w$ is equal to the total number of directed wavelengths $2\lceil K / 3\rceil$. Therefore, $K-p>w=2\lceil K / 3\rceil$, yielding the desired relation

$$
p<K-2\lceil K / 3\rceil \leq\lfloor K / 3\rfloor \text {. }
$$

Lemma 3: In step 3 of the ring RWA algorithm, if no adjacent session pair can be formed among the nonsharing sessions and the new session, then $q \leq\lfloor(K-p) / 2\rfloor$.

Proof: Note that node $i, 1 \leq i \leq N$, is equipped with $k_{i}$ tunable transmitter/receiver pairs. Overall, we have a total of $K$ transmitter/receiver pairs. Each pair of adjacent sessions which share a directed wavelength utilizes one transmitter/receiver pair at some node, one transmitter, and one receiver elsewhere.

Let $p_{i}$ be the number of adjacent session pairs which share a directed wavelength and have node $i$ as a common node. Since an adjacent session pair may have more than one common node, $\sum_{i=1}^{N} p_{i} \geq p$. Let $k_{i}^{\prime}=k_{i}-p_{i}$ denote the number of transmitter/receiver pairs which are not used by those $p_{i}$ adjacent session pairs at node $i$. In addition, let $k_{i}^{t}$ and $k_{i}^{r}$ denote the numbers of nonsharing sessions transmitted and received at node $i$ respectively. It is clear that $k_{i}^{t} \leq k_{i}^{\prime}$ and $k_{i}^{r} \leq k_{i}^{\prime}$.

Since no new adjacent session pair can be formed among the nonsharing sessions, it follows that, at each node $i$, either $k_{i}^{t}=$ 0 or $k_{i}^{r}=0$. Thus, $k_{i}^{t}+k_{i}^{r} \leq k_{i}^{\prime}$. Because each nonsharing session uses one transmitter and one receiver, it follows that

$$
2 q=\sum_{i=1}^{N}\left(k_{i}^{t}+k_{i}^{r}\right) \leq \sum_{i=1}^{N} k_{i}^{\prime}=K-\sum_{i=1}^{N} p_{i} \leq K-p .
$$

Since $q$ is an integer, it follows that $q \leq\lfloor(K-p) / 2\rfloor$.

Proof of algorithm correctness: From the algorithm description, it is clear that we always keep the two desired RWA conditions, i.e. $(i)$ only adjacent sessions share a directed wavelength, and $(i i)$ at most two adjacent sessions share a directed wavelength. In addition, it is clear that at most three lightpath rearrangements are made to support each new session request.

It remains to prove the two claims in step 3. The first claim states that there always exists a new adjacent session pair. We proceed by contradiction. Suppose that no new adjacent session pair can be formed among the nonsharing sessions and the new session. From lemma $3, q \leq\lfloor(K-p) / 2\rfloor$. Since there is no free directed wavelength for the new session in step 3, it follows that the number of wavelengths in use $w$ is equal to the total number of directed wavelengths $2\lceil K / 3\rceil$. Therefore,

$$
p+\lfloor(K-p) / 2\rfloor \geq p+q=w=2\lceil K / 3\rceil .
$$

It follows that

$$
p \geq 2\lceil K / 3\rceil-\lfloor(K-p) / 2\rfloor \geq 2 K / 3-(K-p) / 2,
$$

or equivalently, $p \geq K / 3$, which contradicts the fact that $p<$ $\lfloor K / 3\rfloor$ in step 3 from lemma 2 . Hence, a new adjacent session pair always exists in step 3 .

We now prove the second claim in step 3 that if we need to find a nonsharing session in the opposite ring direction, i.e. the ring direction in which the new adjacent session pair can share a directed wavelength, one always exists. The claim is a direct consequence of lemma 2, i.e. $p<\lfloor K / 3\rfloor$ in step 3. In other words, the number of sharing session pairs is less than the number of directed wavelengths in each ring direction. Since step 2 was not taken, all the other $2\lceil K / 3\rceil-p$ directed wavelengths are taken by nonsharing paths. Therefore, in either ring direction, a directed wavelength with a nonsharing session exists.

The construction of the ring RWA algorithm implies the following theorem.

Theorem 1: For a bidirectional ring with $N$ nodes and $\mathbf{k}-$ allowable traffic, $W_{\mathbf{k}}$ is upper bounded by

$$
W_{\mathbf{k}} \leq\left\lceil\frac{\sum_{i=1}^{N} k_{i}}{3}\right\rceil \text {. }
$$

In addition, there exists, by construction, an on-line RWA algorithm which uses $\left\lceil\left(\sum_{i=1}^{N} k_{i}\right) / 3\right\rceil$ wavelengths in each fiber and requires at most three lightpath rearrangements per new session request.

When $N \geq 7$ and all the $k_{i}$ 's are equal to $k$, it was shown in [17] that $W_{\mathbf{k}}=\lceil k N / 3\rceil$. In this case, the above upper bound is tight. Otherwise, the above upper bound is not necessarily tight and our algorithm may use more than the minimum number of wavelengths. An interesting example is an $N$-node bidirectional ring which contains one hub node, say node 1 , with $k_{1}=N-1$, and the other $N-1$ nodes each with $k_{i}=1$. We shall show below that, in this case, $W_{\mathbf{k}}=\lceil(N-1) / 2\rceil$, which is less than the upper bound $\lceil 2(N-1) / 3\rceil$ from theorem 1 . To do so, we develop an online RWA algorithm which uses $\lceil(N-1) / 2\rceil$ wavelengths and requires at most four lightpath rearrangements per new session request.

\section{A. On-Line RWA for a Single-Hub Bidirectional Ring}

In this subsection, we gives an example scenario for $\mathbf{k}$ allowable traffic in which the ring RWA algorithm does not use the minimum number of wavelengths. Consider a bidirectional ring with $N$ nodes. In particular, node 1 acts as a hub node with $k_{1}=N-1$. In addition, for $2 \leq i \leq N, k_{i}=1$. Note that the non-hub nodes can directly transmit and/or receive wavelengths among themselves.

We first derive a lower bound on the minimum number of wavelengths $W_{\mathbf{k}}$. Consider a cut set corresponding to the two 
links adjacent to the hub node. The maximum traffic across the two fibers leaving from the hub occurs when the hub node transmits $N-1$ wavelengths. Since there are $N-1$ wavelengths travelling on two fibers, one fiber must support at least $\lceil(N-1) / 2\rceil$ wavelengths. Thus, $W_{\mathbf{k}} \geq\lceil(N-1) / 2\rceil$.

We shall prove informally below that $W_{\mathbf{k}} \leq\lceil(N-1) / 2\rceil$, yielding $W_{\mathbf{k}}=\lceil(N-1) / 2\rceil$. A formal proof is based on an on-line RWA algorithm which uses $\lceil(N-1) / 2\rceil$ wavelengths and is given in the appendix.

As in the ring RWA algorithm, the main idea of our RWA involves sharing of a directed wavelength by an adjacent session pair. In addition, we define a special kind of adjacent session pairs as described next. Two sessions form a mutual adjacent session pair if they have two common nodes, i.e. the source node of one session is the destination node of the other session and vice versa. For convenience, we refer to an adjacent session pair which is not mutually adjacent as a nonmutual adjacent session pair. While a nonmutual adjacent session pair can share a directed wavelength in only one ring direction, a mutual adjacent session pair can share a directed wavelength in either ring direction, as shown in figure 5. In particular, the nonmutual adjacent session pair $(1,4)$ and $(4,2)$ can share a directed wavelength in the counterclockwise ring direction, but not in the clockwise ring direction. On the other hand, the mutual adjacent session pair $(2,4)$ and $(4,2)$ can share a directed wavelength in either ring direction.

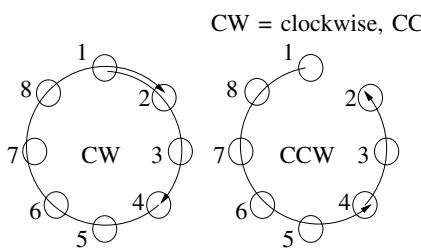

The nonmutual adjacent session pair $(1,4)$ and $(4,2)$ can share a directed wavelength in only one ring direction.

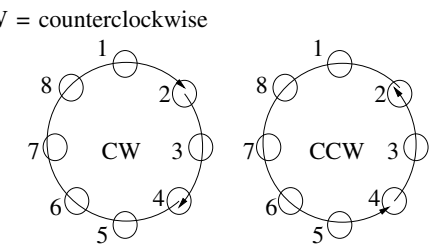

The mutual adjacent session pair $(2,4)$ and $(4,2)$ can share a directed wavelength in either ring direction.
Fig. 5. Supporting a mutual adjacent session pair on a directed wavelength.

We shall refer to an adjacent session pair which has the hub node as a common node as an adjacent session pair at the $h u b$. Our RWA is based on the following two RWA conditions: ( $i$ ) only adjacent session pairs at the hub share a directed wavelength, and (ii) all mutual adjacent session pairs at the hub share a directed wavelength.

Below is our informal proof that $[(N-1) / 2\rceil$ wavelengths are sufficient to support the traffic. We ignore integer rounding in the informal discussion below.

Given a traffic matrix, form all the mutual adjacent session pairs at the hub, but do not assign directed wavelengths for them at this point. Then form all the nonmutual adjacent session pairs at the hub. Let $r$ and $s$ denote the numbers of mutual and nonmutual adjacent session pairs at the hub respectively. Let $t$ be the number of the remaining sessions. Note that we cannot form any new adjacent session pair at the hub among these $t$ sessions.

We first support the $s$ nonmutual adjacent session pairs at the hub on $s$ directed wavelength in the required ring directions. We now show this is always possible. Observe that each non-hub node terminates, i.e. transmits or receives, at most one session in these $s$ adjacent pairs. To see this, note that each non-hub node cannot transmit more than one session since it only has one transmitter. By the same argument, each non-hub node cannot receive more than one session. Moreover, each non-hub node cannot transmit a session and receive a session in these $s$ adjacent pairs simultaneously, or else we can form another mutual adjacent session pair at the hub. It follows that each nonmutual adjacent session pair at the hub is terminated at two non-hub nodes, and no other nonmutual adjacent session pair at the hub is terminated at any of these two nodes. Since there are $N-1$ non-hub nodes, it follows that $s \leq(N-1) / 2$. Since there are $(N-1) / 2$ directed wavelengths available in each ring direction, there are enough wavelengths to support the $s$ session pairs.

We next support the $r$ mutual adjacent session pairs at the hub on any $r$ unused directed wavelengths. We now show this is always possible. Note that each mutual adjacent session pair at the hub is terminated at one distinct non-hub node. From the above discussion, each nonmutual adjacent session pair at the hub is terminated at two distinct non-hub nodes. Since there are $N-1$ non-hub nodes, it follows that $r+2 s \leq N-1$, or equivalently $r \leq(N-1)-2 s$. Since there are $(N-1)-s$ unused directed wavelengths left for this step, the inequality $r \leq(N-1)-2 s$ implies that there are enough directed wavelengths to support the $r$ session pairs.

In the final step, we support the $t$ remaining sessions on any $t$ unused directed wavelengths. We now show this is always possible. Since we cannot form any adjacent session pair at the hub from these $t$ sessions, the hub node can either transmit or receive some or all of these $t$ sessions but not both. Without loss of generality, assume that the hub node transmits none of these $t$ sessions. Consider the transmitters at the non-hub nodes. Each of the $r$ mutual adjacent session pairs at the hub uses one transmitter at some non-hub node. Similarly, each of the $s$ nonmutual adjacent session pairs at the hub uses one transmitter at some non-hub node. Since the hub node does not transmit any of the $t$ remaining sessions, each of the $t$ sessions uses one transmitter at some non-hub node. Since there are $N-1$ non-hub nodes, it follows that $r+s+t \leq N-1$, or equivalently $t \leq(N-1)-r-s$. Since there are $(N-1)-r-s$ unused directed wavelengths left for this step, there are enough directed wavelengths to support the remaining $t$ sessions.

Based on the above main idea of our RWA, we can construct an on-line RWA algorithm which uses $\lceil(N-1) / 2\rceil$ wavelengths in each fiber, is rearrangeably nonblocking, and requires at most four lightpath rearrangements per new session request. We shall refer to this algorithm as the single-hub ring RWA algorithm. We present the algorithm in the appendix but omit the correctness proof which can be found in [20].

\section{TORUS TOPOLOGY}

In this section, we study the RWA problem for $\mathbf{k}$-allowable traffic for a torus topology. We shall consider only the cases in which all the $k_{i}$ 's are equal to some integer $k$. For convenience, we refer to the $\mathbf{k}$-allowable traffic in which all the $k_{i}$ 's are equal to $k$ as symmetric $k$-allowable traffic. The RWA problem 
for general $\mathbf{k}$-allowable traffic remains to be investigated in the future.

Consider an $R \times C$ torus topology with $N$ nodes, where $N=R C$ and $R \geq C$. Let $L_{k}$ be the minimum number of wavelengths which, if provided in each fiber, can support symmetric $k$-allowable traffic given full wavelength conversion at all nodes. Note that $L_{k}$ is the number of wavelengths used to support any traffic matrix in the symmetric $k$-allowable set. Thus, for a specific traffic matrix, we may need fewer wavelengths than in the worst case. We first derive a lower bound on $L_{k}$.

Lemma 4: For an $R \times C$ torus topology with $R \geq C, L_{k} \geq$ $\lceil k(R-1) / 4\rceil$.

Proof: For $R$ even, consider a cut set which separates $R / 2$ consecutive rows of nodes from the other $R / 2$ consecutive rows. Assume a traffic matrix in which each node transmits $k$ wavelengths to a node in the other set. In this traffic, a total of $k R C / 2$ sessions travel from one set of nodes to the other set of nodes on $2 C$ fibers. It follows that one fiber connecting the two sets of nodes must support at least $\left[\frac{k R C / 2}{2 C}\right]=\lceil k R / 4\rceil$ wavelengths. Thus, $L_{k} \geq\lceil k R / 4\rceil$.

For $R$ odd, consider a cut set which separates $(R-1) / 2$ consecutive rows of nodes from the other $(R+1) / 2$ consecutive rows. Assume a traffic matrix in which each node in the smaller set transmits $k$ wavelengths to a node in the other set. In this traffic, a total of $k C(R-1) / 2$ sessions travel from one set of nodes to the other set of nodes on $2 C$ fibers. It follows that one fiber connecting the two sets of nodes must support at least $\left\lceil\frac{k C(R-1) / 2}{2 C}\right\rceil=\lceil k(R-1) / 4\rceil$ wavelengths. Thus, $L_{k} \geq\lceil k(R-1) / 4\rceil$.

In conclusion, for a general (odd or even) positive integer $R, L_{k} \geq\lceil k(R-1) / 4\rceil$.

We shall construct an RWA algorithm which uses $\lceil k R / 2\rceil$ wavelengths in each fiber. The algorithm yields an upper bound on $W_{k}$, the minimum number of wavelengths which, if provided in each fiber, can support symmetric $k$-allowable traffic with no wavelength conversion, i.e. $W_{k} \leq\lceil k R / 2\rceil$. Our upper bound on $W_{k}$ is about twice the value of our lower bound on $L_{k}$.

Define a directed wavelength in the torus topology as follows. Each wavelength consists of an upward directed wavelength and a downward directed wavelength as described next. An upward directed wavelength is directed upwards along any column and to the right along any row, as as illustrated in figure 6a. On the other hand, a downward directed wavelength is directed downwards along any column and to the left along any row.

We shall apply column-first routing where each lightpath travels along the source column and then along the destination row. In addition, each lightpath is supported by no more than one directed wavelength, i.e. if it travels upwards along the source column, then it must travel to the right along the destination row according to the definition of a directed wavelength. The main idea of our RWA algorithm is based on the following observation.

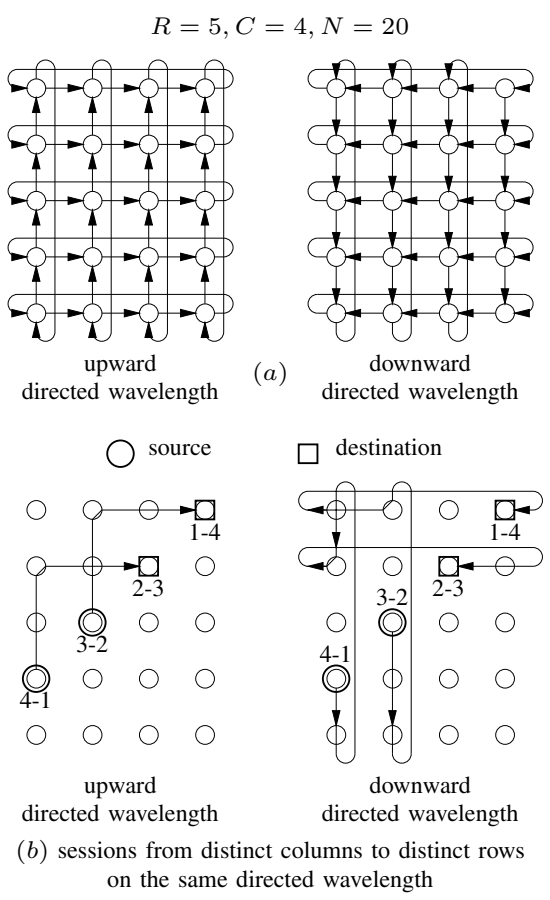

Fig. 6. Directed wavelength and its supported sessions.

Lemma 5: For an $R \times C$ torus topology, under columnfirst routing, a set of sessions from distinct source columns to distinct destination rows can all be supported on a single directed wavelength, which can be either upward or downward directed.

Proof: Since the sessions come from distinct source columns, at most one session utilizes the fibers in a given column. Similarly, since the sessions go to distinct destination rows, at most one session utilizes the fibers in a given row. It follows that there is no wavelength collision on any fiber in the network.

Let $i$ - $j$ denote the node in row $i$ and column $j$. Let $(i$ $j, k-l$ ) denote a session from node $i$-j to node $k-l$. Figure $6 \mathrm{~b}$ illustrates the statement of lemma 5. In particular, there are two sessions (4-1,2-3) and (3-2,1-4) which are transmitted from two distinct source columns to two distinct destination rows. The two sessions can be supported on either an upward or a downward directed wavelength.

We can view the set of sessions from distinct source columns to distinct destination rows as a matching in a bipartite graph. For a given traffic matrix, we can construct the column-to-row bipartite graph, denoted by $\left(\mathcal{V}_{1}, \mathcal{V}_{2}, \mathcal{E}\right)$, as follows. The set of abstract nodes $\mathcal{V}_{1}$ contains $C$ nodes corresponding to the $C$ source columns. The set of abstract nodes $\mathcal{V}_{2}$ contains $R$ nodes corresponding to the $R$ destination rows. In the set of edges $\mathcal{E}$, an edge between node $i$ in $\mathcal{V}_{1}$ and node $j$ in $\mathcal{V}_{2}$ corresponds to a session from a source in column $i$ to a destination in row $j$. Figure 7a-b shows an example of the column-to-row bipartite graph and its traffic matrix. Note that there may be multiple edges between the same pair of nodes.

A matching in a bipartite graph, or in short a bipartite matching, is a subset $\mathcal{M}$ of $\mathcal{E}$ such that no two edges in $\mathcal{M}$ are adjacent. A matching $\mathcal{M}$ is said to saturate the set $\mathcal{V}_{1}$ if, for 


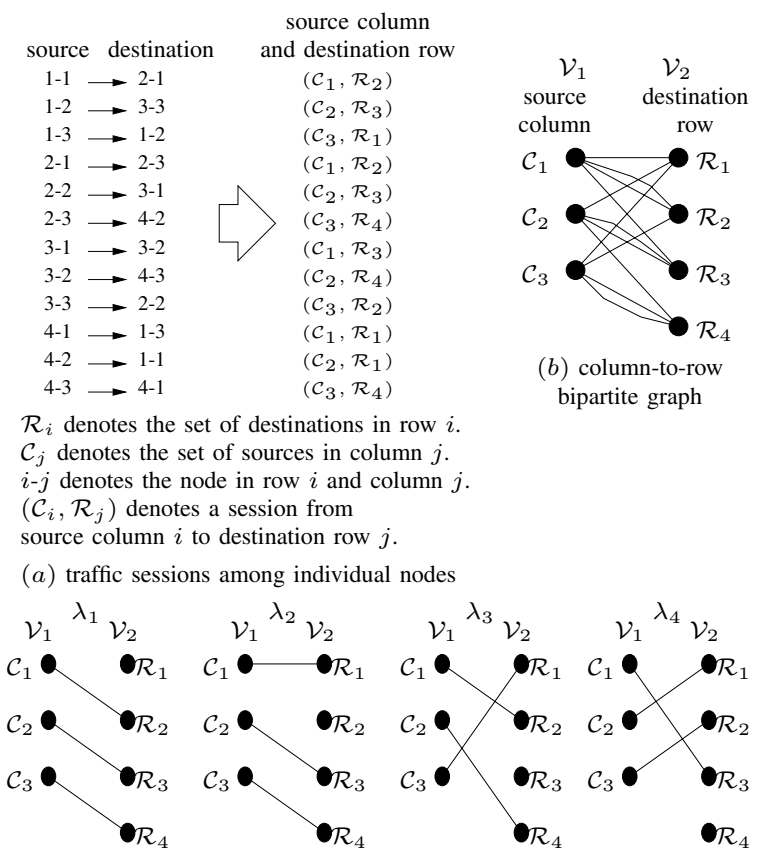

(c) bipartite matchings of specific directed wavelengths

Fig. 7. Column-to-row bipartite graph.

every node in $\mathcal{V}_{1}$, there is an edge in $\mathcal{M}$ incident on that node. A matching $\mathcal{M}$ which saturates the set $\mathcal{V}_{1}$ is called a perfect matching. Figure $7 \mathrm{c}$ shows four different perfect matchings in $\left(\mathcal{V}_{1}, \mathcal{V}_{2}, \mathcal{E}\right)$

Observe that the sessions belonging to a matching in the column-to-row bipartite graph are transmitted from distinct source columns to distinct destination rows. From lemma 5, these sessions can be supported on one directed wavelength using column-first routing. Our algorithm will assign a single bipartite matching to a single directed wavelength. In what follows, we shall refer to the bipartite matching assigned to directed wavelength $\lambda_{1}$ simply as the bipartite matching of $\lambda_{1}$. Figure 7c shows example bipartite matchings of specific directed wavelengths. Note that there are at most $C$ sessions in each matching. We next state a known useful lemma related to bipartite graphs (e.g. [19]).

Lemma 6: In a bipartite graph $\left(\mathcal{V}_{1}, \mathcal{V}_{2}, \mathcal{E}\right)$ with maximum node degree $m$, we can color the edges in $\mathcal{E}$ so that no two adjacent edges have the same color using $m$ colors.

Consider coloring the edges in a bipartite graph $\left(\mathcal{V}_{1}, \mathcal{V}_{2}, \mathcal{E}\right)$ as suggested by lemma 6 . Since no two adjacent edges have the same color, the edges with the same color form a bipartite matching. Thus, we can restate lemma 6 as follows.

Lemma 7: In a bipartite graph $\left(\mathcal{V}_{1}, \mathcal{V}_{2}, \mathcal{E}\right)$ with maximum node degree $m$, the set $\mathcal{E}$ can be partitioned into $m$ disjoint bipartite matchings.

Lemma 7 can be used to argue that $k R$ directed wavelengths are sufficient to support any traffic matrix in the symmtric $k$ allowable set. Given a traffic matrix, we can write down the corresponding column-to-row bipartite graph in which each node has degree at most $k R$. By lemma 7, the set of edges can be partitioned into $k R$ disjoint bipartite matchings. The ses- sions on each bipartite matching can be supported on a single directed wavelength. Therefore, $k R$ directed wavelengths are sufficient to support any symmetric $k$-allowable traffic matrix.

The main idea of our on-line RWA algorithm involves keeping $k R$ disjoint bipartite matchings of $k R$ directed wavelengths such that each traffic session corresponds to an edge in one bipartite matching. When a session departs, we simply remove its corresponding lightpath from the network. When a new session, say $\left(\mathcal{C}_{i}, \mathcal{R}_{j}\right)$, arrives, we find one directed wavelength which is not used by any source in column $i$, and one directed wavelength which is not used by any destination in row $j$. If the two directed wavelength are the same, we can support the new session without any lightpath rearrangement. Otherwise, we rearrange some existing lightpaths on the two directed wavelengths to support the new session. The following lemma makes the above discussion concrete and states an upper bound on the number of lightpath rearrangements.

Lemma 8: In a bipartite graph $\left(\mathcal{V}_{1}, \mathcal{V}_{2}, \mathcal{E}\right)$ with $\left|\mathcal{V}_{1}\right|=C \leq$ $\left|\mathcal{V}_{2}\right|$, given a new edge $\left(\mathcal{C}_{i}, \mathcal{R}_{j}\right), \mathcal{C}_{i} \in \mathcal{V}_{1}, \mathcal{R}_{j} \in \mathcal{V}_{2}$, a matching $\mathcal{M}_{1}$ of directed wavelength $\lambda_{1}$ which is not incident on $\mathcal{C}_{i}$, and a matching $\mathcal{M}_{2}$ of directed wavelength $\lambda_{2}$ which is not incident on $\mathcal{R}_{j}$, there exist two disjoint bipartite matchings which cover all the edges in $\mathcal{M}_{1}$ and $\mathcal{M}_{2}$ as well as the new edge $\left(\mathcal{C}_{i}, \mathcal{R}_{j}\right)$.

In addition, these two disjoint bipartite matchings can be assigned to $\lambda_{1}$ and $\lambda_{2}$ so that the number of lightpath rearrangements is at most $C-1$.

Proof: Consider the bipartite graph $\left(\mathcal{V}_{1}, \mathcal{V}_{2}, \mathcal{E}^{\prime}\right)$ whose set of edges $\mathcal{E}^{\prime}$ contains all of the edges in $\mathcal{M}_{1}$ and $\mathcal{M}_{2}$ as well as the new edge $\left(\mathcal{C}_{i}, \mathcal{R}_{j}\right)$. Observe that each node has degree at most 2. From lemma 7 with $m=2$, there exist two disjoint bipartite matchings, denoted by $\mathcal{M}_{1}^{\prime}$ and $\mathcal{M}_{2}^{\prime}$, which cover all the edges.

Without loss of generality, assume that $\left(\mathcal{C}_{i}, \mathcal{R}_{j}\right)$ belongs to $\mathcal{M}_{1}^{\prime}$. Let set $\mathcal{P}$ contain the edges in $\mathcal{M}_{1}$ assigned to $\mathcal{M}_{2}^{\prime}$ and the edges in $\mathcal{M}_{2}$ assigned to $\mathcal{M}_{1}^{\prime}$. Let set $\mathcal{Q}$ contain the edges in $\mathcal{M}_{1}$ assigned to $\mathcal{M}_{1}^{\prime}$ and the edges in $\mathcal{M}_{2}$ assigned to $\mathcal{M}_{2}^{\prime}$. Notice that $\mathcal{P}$ and $\mathcal{Q}$ cover all the edges in $\mathcal{M}_{1}$ and $\mathcal{M}_{2}$. Since there are at most $2 C-2$ edges in $\mathcal{M}_{1}$ and $\mathcal{M}_{2}$, it follows that $|\mathcal{P}|+|\mathcal{Q}| \leq 2 C-2$.

If $|\mathcal{P}| \leq C-1$, assigning $\mathcal{M}_{1}^{\prime}$ to $\lambda_{1}$ and $\mathcal{M}_{2}^{\prime}$ to $\lambda_{2}$ yields the desired result that the number of lightpath rearrangements, which is equal to the sum of the number of edges in $\mathcal{M}_{1}$ assigned to $\mathcal{M}_{2}^{\prime}$ and the number of edges in $\mathcal{M}_{2}$ assigned to $\mathcal{M}_{1}^{\prime}$, is at most $C-1$. Otherwise, it is true that $|\mathcal{Q}| \leq C-1$. In this case, assigning $\mathcal{M}_{1}^{\prime}$ to $\lambda_{2}$ and $\mathcal{M}_{2}^{\prime}$ to $\lambda_{1}$ yields the desired result.

The following is our on-line RWA algorithm for a torus topology with symmetric $k$-allowable traffic. The algorithm uses $\lceil k R / 2\rceil$ wavelengths in each fiber, is rearrangeably nonblocking, and requires at most $C-1$ lightpath rearrangements per new session request. We shall refer to this algorithm as the torus RWA algorithm

Torus RWA Algorithm: (Use $\lceil k R / 2\rceil$ wavelengths.)

Session termination: When a session terminates, simply remove its associated lightpath from the network without any 
further lightpath rearrangement.

Session arrival: When a session arrives and it is allowable, proceed as follows. Let $i$ and $j$ denote the source column and the destination row of the new session.

Step 1: If there is a directed wavelength, denoted by $\lambda_{0}$, which is used by neither a source in column $i$ nor a destination in row $j$, then assign the new session to $\lambda_{0}$, and use column-first routing. In this case, no lightpath rearrangement is required. Otherwise, proceed to step 2.

Step 2: Find a directed wavelength, denoted by $\lambda_{1}$, which is not used by any source in column $i$, i.e. its bipartite matching is not incident on $\mathcal{C}_{i}$, and another directed wavelength, denoted by $\lambda_{2}$, which is not used by any destination in row $j$, i.e. its bipartite matching is not incident on $\mathcal{R}_{j}$. We claim and shall prove shortly that $\lambda_{1}$ and $\lambda_{2}$ exist.

Modify the RWA of only the sessions on $\lambda_{1}$ and $\lambda_{2}$. Construct the column-to-row bipartite graph $\left(\mathcal{V}_{1}, \mathcal{V}_{2}, \mathcal{E}^{\prime}\right)$ in which the set of edges $\mathcal{E}^{\prime}$ contains the bipartite matchings of $\lambda_{1}$ and $\lambda_{2}$ as well as the new edge $\left(\mathcal{C}_{i}, \mathcal{R}_{j}\right)$. Notice that $\left|\mathcal{V}_{1}\right|=C \leq R=\left|\mathcal{V}_{2}\right|$ and each abstract node has degree at most 2. From lemma 8 , the set $\mathcal{E}^{\prime}$ can be partitioned into two disjoint bipartite matchings. In addition, lemma 8 tells us that the two matchings can be assigned to $\lambda_{1}$ and $\lambda_{2}$ such that at most $C-1$ existing lightpaths need to be rearranged.

Proof of algorithm correctness: It remains to prove the claim in step 2, which states that either there is a directed wavelength which is used by neither a source in column $i$ nor a destination in row $j$, or $\lambda_{1}$ and $\lambda_{2}$ as defined in step 2 must exist. We shall prove the existence of $\lambda_{1}$. Similar arguments can be used to prove the existence of $\lambda_{2}$. Since the new session is allowable, there are at most $k R-1$ sessions transmitted from source column $i$. Since there are $2\lceil k R / 2\rceil$ directed wavelengths, the number of directed wavelengths available for a session transmitted from source column $i$ is at least

$$
2\lceil k R / 2\rceil-(k R-1) \geq k R-(k R-1) \geq 1 .
$$

Therefore, $\lambda_{1}$ always exists.

Although we concentrate on an $R \times C$ torus topology with $R \geq C$, similar results can be obtained for an $R \times C$ torus topology with $R \leq C$ by reversing the roles of rows and columns. We summarize the results in this section in the following theorem.

Theorem 2: For an $R \times C$ torus network with symmetric $k$-allowable traffic, $W_{k}$ is bounded by

$$
\left\lceil\frac{k(\max (R, C)-1)}{4}\right\rceil \leq L_{k} \leq W_{k} \leq\left\lceil\frac{k \max (R, C)}{2}\right\rceil .
$$

In addition, there exists, by construction, an on-line RWA algorithm which uses $\lceil k \max (R, C) / 2\rceil$ wavelengths in each fiber and requires at most $\min (R, C)-1$ lightpath rearrangements per new session request.

As a comparison, when $\min (R, C)=1$, we have a bidirectional ring with $N$ nodes, where $N=R C$. The torus RWA algorithm in this section uses $\lceil k N / 2\rceil$ wavelengths in each fiber while the ring RWA algorithm specialized for the ring topology uses $\lceil k N / 3\rceil$ wavelengths. Hence, while the torus RWA algorithm is more general, it uses more wavelengths than the minimum required for the ring topology.

We observe from the two algorithms that the number of lightpath rearrangements per new session request is related to the number of lightpaths supported on a single directed wavelength. For a bidirectional ring, up to two lightpaths are supported on a single directed wavelength. Since the ring RWA algorithm modifies only the RWA of the sessions on at most three directed wavelengths, it follows that the number of lightpath rearrangements depends on neither the number of nodes $N$ nor the amount of traffic $\mathbf{k}$. For a torus topology, up to $C$ lightpaths are supported on a single directed wavelength. Since the torus RWA algorithm modifies only the RWA of the sessions on at most two directed wavelengths, it follows that the number of lightpath rearrangements depends on the dimension of the network $C$ but not on the amount of traffic k.

\section{CONCLUSION}

We developed an on-line routing and wavelength assignment (RWA) algorithm a for WDM bidirectional ring with $N$ nodes to support $\mathrm{k}$-allowable traffic in a rearrangeably nonblocking fashion. The algorithm uses $\left\lceil\left(\sum_{i=1}^{N} k_{i}\right) / 3\right\rceil$ wavelengths in each ring direction and requires at most three lightpath rearrangements per new session request regardless of the number of nodes $N$ and the amount of traffic $\mathbf{k}$.

The developed algorithm implies the upper bound on $W_{\mathbf{k}}$, i.e. $W_{\mathbf{k}} \leq\left\lceil\left(\sum_{i=1}^{N} k_{i}\right) / 3\right\rceil$. The bound is tight for the case in which $N \geq 7$ and all the $k_{i}$ 's are equal to some positive integer $k$. In addition, we observed that, for $N \geq 7$ and a fixed value of $\sum_{i=1}^{N} k_{i}$ equal to $k N$ for some positive integer $k$, the case in which all the $k_{i}$ 's are equal yields the maximum value of $W_{\mathbf{k}}$.

We also developed an on-line RWA algorithm for an $R \times C$ torus topology to support $\mathbf{k}$-allowable traffic, where all the $k_{i}$ 's are equal to some positive integer $k$, in a rearrangeably nonblocking fashion. The algorithm uses $\lceil k \max (R, C) / 2\rceil$ wavelengths in each fiber, which is shown to be approximately two times a lower bound obtained by assuming full wavelength conversion at all nodes. In addition, the algorithm requires at $\operatorname{most} \min (R, C)-1$ lightpath rearrangements per new session request regardless of the amount of traffic $k$. Our future goal is to develop an on-line RWA algorithm for arbitrary mesh topologies. We hope that our analytical approaches in this paper can be used in the development of such an algorithm.

\section{APPENDIX}

\section{Single-Hub Ring RWA AlgORITHM}

In the algorithm below, we maintain two RWA conditions at all time: $(i)$ only adjacent session pairs at the hub share a directed wavelength, and ( $i$ i) all mutual adjacent session pairs at the hub share a directed wavelength.

Single-Hub Ring RWA Algorithm: (Use $\lceil(N-1) / 2\rceil$ wavelengths and perform at most four lightpath rearrangements per new session request.) 
Session termination: When a session terminates, simply remove its associated lightpath from the ring without any further lightpath rearrangement.

Session arrival: When a session arrives and the resultant traffic matrix is still $\mathrm{k}$-allowable, proceed as follows.

Step 1: If the new session, denoted by $u$, can form a mutual adjacent session pair at the hub with some existing session, denoted by $x$, there are two possibilities.

(1a) If $x$ is not sharing its directed wavelength, assign the mutual adjacent session pair $u$ and $x$ to share this directed wavelength. In this case, no lightpath rearrangement is required.

(1b) If $x$ is sharing a directed wavelength with another existing session, denoted by $y$, then $x$ and $y$ are not mutually adjacent at the hub, or else $u$ and $x$ cannot be mutually adjacent at the hub. Remove $y$ from its directed wavelength and assign the mutual adjacent session pair $u$ and $x$ to share the directed wavelength of $y$.

If there is a free directed wavelength, use it to support $y$. In this case, one lightpath rearrangement is made. Otherwise, we claim that $y$ can form another adjacent session pair at the hub with some nonsharing session, denoted by $z$. Note that $y$ and $z$ cannot be mutually adjacent at the hub, or else they would have shared a directed wavelength.

If the directed wavelength of $z$ can support $y$, assign $y$ and $z$ to share this directed wavelength. In this case, one lightpath rearrangement is made. Otherwise, we claim that there must exist either a nonsharing session or a mutual adjacent session pair in the opposite ring direction. In the case of a nonsharing session in the opposite ring direction, we remove that nonsharing session and support $y$ and $z$ on its directed wavelength. The removed nonsharing session can then be supported on the directed wavelength of $z$. In this case, a total of three lightpath rearrangements are made. In the case of a mutual adjacent session pair in the opposite ring direction, we remove that mutual adjacent session pair and support $y$ and $z$ on their directed wavelength. The removed mutual adjacent session pair can then be supported on the directed wavelength of $z$. In this case, a total of four lightpath rearrangements are made.

Step 2: If $u$ cannot form a mutual adjacent session pair at the hub with any existing session and there is a free directed wavelength, use a free directed wavelength to support $u$. In this case, no lightpath rearrangement is made.

Step 3: If $u$ cannot form a mutual adjacent session pair at the hub with any existing session and there is no free directed wavelength, we claim that, among nonsharing sessions and $u$, a nonmutual adjacent session pair at the hub can be formed. Denote this session pair by $y$ and $z$. There are two possibilities.

(3a) If $u$ is in the session pair, i.e. $y=u$ or $z=u$, assume without loss of generality that $y=u$. If the directed wavelength of $z$ can support $y$, assign $y$ and $z$ to share this directed wavelength. In this case, no lightpath rearrangement is required. Otherwise, we claim there must exist either a nonsharing session or a mutual adjacent session pair in the opposite ring direction. In the case of a nonsharing session in the opposite ring direction, we remove that nonsharing session and support $y$ and $z$ on its directed wavelength. The removed nonsharing session can then be supported on the directed wavelength of $z$. In this case, a total of two lightpath rearrangements are made. In the case of a mutual adjacent session pair in the opposite ring direction, we remove that mutual adjacent session pair and support $y$ and $z$ on their directed wavelength. The removed mutual adjacent session pair can then be supported on the directed wavelength of $z$. In this case, a total of three lightpath rearrangements are made.

(3b) If $u$ is not in the session pair, then $y \neq u$ and $z \neq u$. If the directed wavelength of either $y$ or $z$ can support the session pair, assign $y$ and $z$ to share this directed wavelength. This sharing frees one directed wavelength on which $u$ can be supported. In this case, one lightpath rearrangement is made. Otherwise, we claim that there must exist either a nonsharing session or a mutual adjacent session pair in the opposite ring direction. In the case of a nonsharing session in the opposite ring direction, we remove that nonsharing session and support $y$ and $z$ on its directed wavelength. The removed nonsharing session and the new session can then be supported on the directed wavelengths of $y$ and $z$. In this case, a total of three lightpath rearrangements are made. In the case of a mutual adjacent session pair in the opposite ring direction, we remove that mutual adjacent session pair and support $y$ and $z$ on their directed wavelength. The removed mutual adjacent session pair and the new session can then be supported on the directed wavelengths of $y$ and $z$. In this case, a total of four lightpath rearrangements are made.

The proof of algorithm correctness can be found in [20].

\section{REFERENCES}

[1] D. Banerjee and B. Mukherjee, "A practical approach for routing and wavelength assignment in large wavelength-routed optical networks," IEEE JSAC, vol. 14, no. 5, pp. 903-908, June 1996.

[2] R. Ramaswami and K.N. Sivarajan, "Design of logical topologies for wavelength-routed optical networks," IEEE JSAC, vol. 14, no. 5, pp. 840851, June 1996.

[3] I. Chlamtac, A. Ganz, and G. Karmi, "Lightpath communications: an approach to high bandwidth optical WAN's," IEEE Trans. on Comm., vol. 40, no. 7, pp. 1171-1182, July 1992.

[4] R. Ramaswami and K.N. Sivarajan, "Routing and wavelength assignment in all-optical networks," IEEE/ACM Trans. on Networking, vol. 3, no. 5, pp. 489-500, October 1995.

[5] Z. Zhang and A.S. Acampora, "A heuristic wavelength assignment algorithm for multihop WDM networks with wavelength routing and wavelength reuse," IEEE/ACM Trans. on Networking, vol. 3, no. 3, pp. 281-288, June 1995.

[6] C. Chen and S. Banerjee, "A new model for optimal routing and wavelength assignment in wavelength division multiplexed optical networks," IEEE INFOCOM 1996, pp. 164-171, April 1996.

[7] S. Banerjee, J. Yoo, and C. Chen, "Design of wavelength-routed optical networks for packet switched traffic," Jour. of Lightwave Tech., vol. 15, no. 9, pp. 1636-1646, September 1997.

[8] K.C. Lee and V.O.K. Li, "A wavelength rerouting algorithm in widearea all-optical networks," Jour. of Lightwave Tech., vol. 14, no. 6, pp. 1218-1229, June 1996.

[9] B. Mukherjee et al., "Some principles for designing a wide-area WDM optical network,"IEEE/ACM Trans. on Networking, vol. 4, no. 5, pp. 684696, October 1996

[10] A. Birman, "Computing approximate blocking probabilities for a class of all-optical networks," IEEE JSAC, vol. 14, no. 5, pp. 852-857, June 1996. 
[11] S. Subramaniam, M. Azizoglu, and A.K. Somani, "All-optical networks with sparse wavelength conversion," IEEE/ACM Trans. on Networking, vol. 4, no. 4, pp. 544-557, August 1996.

[12] R.A. Barry and P.A. Humblet, "Models of blocking probability in alloptical networks with and without wavelength changers," IEEE JSAC, vol. 14, no. 5, pp. 858-867, June 1996.

[13] L. Li and A.K. Somani, "Dynamic wavelength routing using congestion and neighborhood information," IEEE/ACM Trans. on Networking, vol. 7, no. 5, pp. 779-786, October 1999.

[14] Y. Zhu, G.N. Rouskas, and H.G. Perros, "A path decomposition algorithm for computing blocking probabilities in wavelength routing networks," IEEE/ACM Trans. on Networking, vol. 8, no. 6, pp. 747-762, December 2000.

[15] R.K. Pankaj, Architectures for Linear Lightwave Networks, MIT Ph.D. Thesis, September 1992.

[16] O. Gerstel, G. Sasaki, S. Kutten, and R. Ramaswami, "Worst-case analysis of dynamic wavelength allocation in optical networks," IEEE/ACM Trans. on Networking, vol. 7, no. 6, pp. 833-845, December 1999.

[17] A. Narula-Tam, P.J. Lin, and E.H. Modiano, "Efficient routing and wavelength assignment for reconfigurable WDM networks," IEEE JSAC, vol. 20, no. 1, pp. 75-88, January 2002.

[18] P. Saengudomlert, E.H. Modiano, and R.G. Gallager, "An on-line routing and wavelength assignment algorithm for dynamic traffic in a WDM bidirectional ring," Joint Conference on Information Sciences, pp.13311334, March 2002.

[19] C. Berge, Graphs. North-Holland, 1985.

[20] P. Saengudomlert, Architectural Study of High-Speed Networks with Optical Bypassing, MIT Ph.D. Thesis, September 2002. 